\title{
On summing to arbitrary real numbers
}

\author{
Jaroslav Hančl, Jan Šustek, Radhakrishnan Nair, \\ Pavel Rucki and Dmitry Bodyagin
}

\begin{abstract}
Jaroslav Hančl completed his studies in mathematics at Charles University in Prague in 1984. Presently he holds a position at the University of Ostrava. His main field of research is diophantine approximation.

Jan Šustek is a Ph.D. student at the Department of Mathematics of the University of Ostrava working in diophantine approximation.

Radhakrishnan Nair received his Ph.D. at the University of Warwick in 1986. Presently he holds a position at the University of Liverpool. His main field of research are questions on distributions in number theory.

Pavel Rucki completed his studies in mathematics at the University of Ostrava in 2006. Presently he is a postdoctoral fellow at the same university. His main field of research is diophantine approximation.

Dmitry Bodyagin is a postgraduate student at the Institute of Mathematics of the Belarusian Academy of Sciences. His main field of research is diophantine approximation.
\end{abstract}

Following Erdős [1] we say a sequence $\left\{a_{n}\right\}_{n=1}^{\infty}$ is irrational if the set $\left\{\sum_{n \geq 1} \frac{1}{a_{n} c_{n}} \mid c_{n} \in\right.$ $\mathbb{N}\}$, which we refer to henceforth as its expressible set, contains no rational numbers. In [1] it is shown that if $\lim _{n \rightarrow \infty} a_{n}^{1 / 2^{n}}=\infty$ and $a_{n} \in \mathbb{N}$ for all $n \in \mathbb{N}$ then $\sum_{n \geq 1} a_{n}^{-1}$ is an irrational number. From this Erdős deduces that the sequence $\left\{2^{2^{n}}\right\}_{n=1}^{\infty}$ is an irrational sequence. Thus its expressible set contains no rational numbers. In [2] it is shown that if $a_{n} \in \mathbb{R}^{+}$for all $n \in \mathbb{N}$ and $\limsup _{n \rightarrow \infty} \frac{1}{n} \log _{2} \log _{2} a_{n}<1$ then the expressible set of the sequence $\left\{a_{n}\right\}_{n=1}^{\infty}$ contains an interval. It seems to be the case that in general finding the expressible set for the sequence $\left\{a_{n}\right\}_{n=1}^{\infty}$ is not easy.

Ein interessantes zahlentheoretisches Problem ist die Frage nach der Rationalität des Werts einer konvergenten Reihe reeller Zahlen. An diese Fragestellung anknüpfend nennen wir mit P. Erdős eine Folge $\left\{a_{n}\right\}_{n=1}^{\infty}$ reeller Zahlen irrational, falls die Menge $E=\left\{\sum_{n=1}^{\infty} 1 /\left(a_{n} c_{n}\right) \mid c_{n} \in \mathbb{N}\right\}$ keine rationale Zahl enthält. In der vorliegenden Arbeit beweisen die Autoren für den Fall, dass die Reihe $\sum_{n=1}^{\infty} 1 / a_{n}$ bedingt konvergent ist, dass die Menge $E$ jeweils die gesamte reelle Zahlengerade ausschöpft. 
In this paper we give conditions on $\left\{a_{n}\right\}_{n=1}^{\infty}$ to ensure that its expressible set is equal to $\mathbb{R}$. We prove the following:

Theorem 1. Let $\left\{a_{n}\right\}_{n=1}^{\infty}$ be a sequence of nonzero real numbers such that the series $\sum_{n=1}^{\infty} \frac{1}{a_{n}}$ is conditionally convergent. Then its expressible set is equal to $\mathbb{R}$.

A series is conditionally convergent if it is convergent but the series of the absolute values of its terms is not. Theorem 1 is an immediate consequence of the following more general theorem.

Theorem 2. Let $\left\{a_{n}\right\}_{n=1}^{\infty}$ be a sequence of nonzero real numbers such that the series $\sum_{n=1}^{\infty} \frac{1}{a_{n}}$ is conditionally convergent. Then for every pair $\alpha, \beta$ of real numbers with $\alpha \leq \beta$ there exists a sequence $\left\{c_{n}\right\}_{n=1}^{\infty}$ of positive integers such that

$$
\alpha=\liminf _{N \rightarrow \infty} \sum_{n=1}^{N} \frac{1}{a_{n} c_{n}} \quad \text { and } \quad \beta=\limsup _{N \rightarrow \infty} \sum_{n=1}^{N} \frac{1}{a_{n} c_{n}} .
$$

For the proof of Theorem 2 we need the following two lemmas.

Lemma 1. Let $\left\{a_{n}\right\}_{n=1}^{\infty}$ be a sequence of nonzero real numbers such that the series $\sum_{n=1}^{\infty} \frac{1}{a_{n}}$ is conditionally convergent. Then for every real number $A \geq 0$ and every integer $N \geq 0$ there exist a number $K \in \mathbb{N}$ and numbers $c_{N+1}, \ldots, c_{N+K} \in \mathbb{N}$ such that

$$
\sum_{n=N+1}^{N+K} \frac{1}{a_{n} c_{n}} \in\left(A, A+\frac{1}{a_{N+K}}\right] \text {. }
$$

Proof. Define $\mathcal{P}=\left\{n \mid a_{n}>0\right\}$ and $\mathcal{N}=\left\{n \mid a_{n}<0\right\}$. The series $\sum_{n=1}^{\infty} \frac{1}{a_{n}}$ is conditionally convergent, hence

$$
\sum_{\substack{n=N+1 \\ n \in \mathcal{P}}}^{\infty} \frac{1}{a_{n}}=\infty
$$

This implies that there exists a positive integer $K$ such that

$$
\sum_{\substack{n=N+1 \\ n \in \mathcal{P}}}^{N+K-1} \frac{1}{a_{n}} \leq A \quad \text { and } \quad \sum_{\substack{n=N+1 \\ n \in \mathcal{P}}}^{N+K} \frac{1}{a_{n}}>A .
$$

The fact that

$$
0<\sum_{\substack{n=N+1 \\ n \in \mathcal{P}}}^{N+K} \frac{1}{a_{n}}-\sum_{\substack{n=N+1 \\ n \in \mathcal{P}}}^{N+K-1} \frac{1}{a_{n}}=\frac{1}{a_{N+K}}
$$

immediately gives

$$
s=\sum_{\substack{n=N+1 \\ n \in \mathcal{P}}}^{N+K} \frac{1}{a_{n}} \in\left(A, A+\frac{1}{a_{N+K}}\right]
$$


Now consider two cases:

(1) Assume that $\mathcal{N} \cap\{N+1, \ldots, N+K\}=\emptyset$. In this case put $c_{n}=1$ for every $n=N+1, \ldots, N+K$ and the result follows.

(2) Now suppose that

$$
r=\sum_{\substack{n=N+1 \\ n \in \mathcal{N}}}^{N+K} \frac{1}{a_{n}}<0
$$

Put $C=\left[\frac{r}{A-s}\right]+1$. Then

$$
0>\sum_{\substack{n=N+1 \\ n \in \mathcal{N}}}^{N+K} \frac{1}{C a_{n}}=\frac{1}{C} \sum_{\substack{n=N+1 \\ n \in \mathcal{N}}}^{N+K} \frac{1}{a_{n}}>\frac{A-s}{r} \cdot r=A-s .
$$

Hence the result follows by taking $c_{n}=1$ for $n \in\{N+1, \ldots, N+K\} \cap \mathcal{P}$ and $c_{n}=C$ for $n \in\{N+1, \ldots, N+K\} \cap \mathcal{N}$.

Lemma 2. Let $\left\{a_{n}\right\}_{n=1}^{\infty}$ be a sequence of nonzero real numbers such that the series $\sum_{n=1}^{\infty} \frac{1}{a_{n}}$ is conditionally convergent. Then for every real number $A \leq 0$ and every integer $N^{2} \geq 0$ there exist a number $K \in \mathbb{N}$ and numbers $c_{N+1}, \ldots, c_{N+K} \in \mathbb{N}$ such that

$$
\sum_{n=N+1}^{N+K} \frac{1}{a_{n} c_{n}} \in\left[A-\left|\frac{1}{a_{N+K}}\right|, A\right) .
$$

Proof. Using the transformation $a_{n} \mapsto-a_{n}$ and Lemma 1 we obtain Lemma 2.

Proof of Theorem 2. In the following we set

$$
S_{k}=\sum_{n=1}^{k} \frac{1}{a_{n} c_{n}}
$$

If $\beta \geq 0$ then putting $A=\beta$ and $N=0$ into Lemma 1 we obtain a number $K$ and a sequence $\left\{c_{n}\right\}_{n=1}^{K}$ such that

$$
S_{K} \in\left(\beta, \beta+\frac{1}{a_{K}}\right] \text {. }
$$

Then set $N_{0}=0$ and $N_{1}=K$.

Similarly, if $\beta<0$ then $\alpha<0$, and putting $A=\alpha$ and $N=0$ into Lemma 2 we get $K$ and $\left\{c_{n}\right\}_{n=1}^{K}$ with

$$
S_{K} \in\left[\alpha-\left|\frac{1}{a_{K}}\right|, \alpha\right) .
$$

Then set $N_{0}=K$. 
Now we will construct the sequence $\left\{c_{n}\right\}_{n=1}^{\infty}$ by induction. Consider two cases:

(1) Suppose that we have constructed the sequence $\left\{N_{m}\right\}_{m=0}^{2 t+1}, t \in \mathbb{N}_{0}$, with

$$
S_{N_{2 t+1}} \in\left(\beta, \beta+\frac{1}{a_{N_{2 t+1}}}\right] \text {. }
$$

Lemma 2 implies that there exist $K$ and $\left\{c_{n}\right\}_{n=N_{2 t+1}+1}^{N_{2 t+1}+K}$ such that

$$
\sum_{n=N_{2 t+1}+1}^{N_{2 t+1}+K} \frac{1}{a_{n} c_{n}} \in\left[\alpha-S_{N_{2 t+1}}-\left|\frac{1}{a_{N_{2 t+1}+K}}\right|, \alpha-S_{N_{2 t+1}}\right) .
$$

Let $N_{2 t+2}=N_{2 t+1}+K$. Then we have

$$
S_{N_{2 t+2}} \in\left[\alpha-\left|\frac{1}{a_{N_{2 t+2}}}\right|, \alpha\right) .
$$

(2) Suppose that we have constructed the sequence $\left\{N_{m}\right\}_{m=0}^{2 t}, t \in \mathbb{N}_{0}$, with

$$
S_{N_{2 t}} \in\left[\alpha-\left|\frac{1}{a_{N_{2 t}}}\right|, \alpha\right) \text {. }
$$

Lemma 1 implies that there exist $K$ and $\left\{c_{n}\right\}_{n=N_{2 t}+1}^{N_{2 t}+K}$ such that

$$
\sum_{n=N_{2 t}+1}^{N_{2 t}+K} \frac{1}{a_{n} c_{n}} \in\left(\beta-S_{N_{2 t}}, \beta-S_{N_{2 t}}+\frac{1}{a_{N_{2 t}+K}}\right] .
$$

Let $N_{2 t+1}=N_{2 t}+K$. Then we have

$$
S_{N_{2 t+1}} \in\left(\beta, \beta+\frac{1}{a_{N_{2 t+1}}}\right] .
$$

Using alternatively cases (1) and (2) we construct the whole sequence $\left\{c_{n}\right\}_{n=1}^{\infty}$. From the construction it follows that

- $\quad \alpha-\left|\frac{1}{a_{k}}\right| \leq S_{k} \leq \beta+\left|\frac{1}{a_{k}}\right| \quad$ for every $k \geq N_{1}$,

- $S_{N_{2 t}}<\alpha$ for every $t \in \mathbb{N}$,

- $\quad S_{N_{2 t+1}}>\beta \quad$ for every $t \in \mathbb{N}_{0}$.

The series $\sum_{n=1}^{\infty} \frac{1}{a_{n}}$ is conditionally convergent, hence $\frac{1}{a_{n}} \rightarrow 0$. This implies that

$$
\lim _{t \rightarrow \infty} S_{N_{2 t}}=\alpha \text { and } \quad \lim _{t \rightarrow \infty} S_{N_{2 t+1}}=\beta
$$

and the result follows. 


\section{References}

[1] Erdôs, P.: Some problems and results on the irrationality of the sum of infinite series. J. Math. Sci. 10 (1975), 1-7.

[2] Hančl, J.: Expression of Real Numbers With the Help of Infinite Series. Acta Arith. LIX.2, (1991), 97-104.

Jaroslav Hančl, Jan Šustek

Department of Mathematics and Institute for

Research and Applications of Fuzzy Modeling

University of Ostrava

30. dubna 22

70103 Ostrava 1, Czech Republic

e-mail: hancl@osu.cz, jan.sustek@seznam.cz

Radhakrishnan Nair

Mathematical Sciences

Peach Street

Liverpool L69 7ZL, U.K.

e-mail: nair@liverpool.ac.uk

Pavel Rucki

Department of Mathematics and Didactics

University of Ostrava

Dvořákova 7

70103 Ostrava 1, Czech Republic

e-mail: pavel.rucki@seznam.cz

Dmitry Bodyagin

Department of Theory of Numbers

Institute of Mathematics

National Academy of Sciences of Belarus

Surganov str. 11

220072 Minsk, Belarus

e-mail: bodiagin@mail.ru

The paper was supported by the grants no. 201/04/0381, 201/07/0191, and MSM6198898701 